\title{
The Multivocality of Health Merits a Closer Look at Habilitation of the Disenfranchised Worker
}

Linda Marie Small, MA, OTR

Founder

Progressive Steps: Concepts in Rehabilitation, Inc. Tucson, Arizona

The word healthy can be traced back through Old German to its common origins with the words hale and whole. The word hale brings to mind thoughts of endurance, fitness, strength, and physiologically combined bodily function. Whole draws upon images of integration of mental and physical processes. Whole signals a harmonious interplay between the individual and society.

Being healthy, then, is a deceptive proposition in that it entails more that just biologically operative flesh. Being healthy is, in great measure, derived from epoch-specific social comparison. Individuals in every era perceive the culturally correct body-being, form and functioning, through comparison with other body-beings within their respective social networks. Health is part of our construction of the self. It entails perceptions of "normal" in bodily form and in social functioning that are subculture-specific norms of being and of doing. "Healthy" is a socially derived notion.

Medicine, on the other hand, has long looked at "function" as a measure of health. Functional skills support independent living, participation in society, and quality of life. Functional assessments are used to determine disability ratings for welfare and insurance benefits; but health is more than mere functional ability, more than a repertoire of performance skills. The individual's relative state of health can be gleaned from their purposeful occupation within society. Purposeful occupation, then, not potential ability alone, is a more certain measure of individual health.

Despite biologically operative flesh and functional potential, the depressed person, as an example, is not considered a model of health or function largely because he/she is unable or unwilling to become purposefully and adequately engaged in activity. Commonly, even routine activities such as appropriate grooming and bathing may not be adequately undertaken. In contrast, a person with a physical disability, such as blindness, may not be perceived as unhealthy because he/she is purposefully engaged in the daily routines of living. The person is purposefully engaged in leisure and work, albeit by compensating for the lack of vision in ways the sighted may not employ. Therefore, the individual, although blind (disabled), is healthy.

In U.S. culture, purposeful occupation is largely conceived of as income-producing work for adult members of society. With this conception of purposeful occupation as work, work can be seen as health enabling. Work provides for the engagement of functional skills and forms a major component of the individual's personal and social identity. Herein, a question arises as to whether the disenfranchised are in fact healthy.

To illustrate this concept of purposeful occupation, let me describe my experience of Sawyer Brown's pop musical hit, "The Cafe Down At The Corner." As an itinerate therapist, I often stopped off in neighboring towns to prepare for that afternoon's teaching assignment. As I gazed about at 
midmorning, I saw a number of the town's residents drinking coffee. I, as an evidently employed person (I had briefcase and tools in hand), attracted immediate notice in this region of high unemployment.

The town residents would not have been my medically inspired notion of disabled, as they had no immediately evident biological impairments or discernible loss of social skills. Yet, the life stories I heard and continue to hear indicate a severe deficit of purposeful occupation. Many lacked salable work skills in this technologically fast-paced era. They also described inadequate housing, broken family ties, poor social network exchange, violence, and vague physical complaints that could be categorized as not "feeling well."

My observations of growing unemployment and the failure of many to regain monetarily productive work and adequate social networks have made me reexamine the meaning of health and purposeful occupation. Here, it is well to ponder the possible habilitative roles that therapy might play in intervention or reengagement strategies with the economically disenfranchised. Certainly there is a need for performance skills such as resume writing, job application how-to, and work skills fitting the technology of today. But function and health, as portrayed herein, are culturally inscribed in the individual's notion of self. A job shift from a manufacturing to a service industry, for instance, may involve cultivating new conceptions of "normal" and new social roles to access emergent subculture groups. Thus, therapeutic intervention may pivot on the acquisition of new social, as well as performance, skills to successfully enter into the rapidly evolving cultural networks of these changing economic times. This would entail a view of function as more than biological integrity or a repertoire of technical skills. Function is purposeful occupation. Purposeful occupation, then, becomes a measure of health that can be therapeutically addressed. 